OITS-664

RU-98-48

BUHEP-98-27

\title{
Non-Perturbative Couplings and Color Superconductivity
}

\author{
Nick Evans* \\ Department of Physics, Boston University, Boston, MA 02215. \\ Stephen D.H. Hsu ${ }^{\dagger}$ \\ Department of Physics, University of Oregon, Eugene OR 97403-5203. \\ Myckola Schwetz ${ }^{\ddagger}$ \\ Department of Physics and Astronomy, Rutgers University, Piscataway NJ 08855-0849.
}

\section{October 1998}

\begin{abstract}
Quark matter at sufficiently high density exhibits color superconductivity, due to attractive gluonic interactions. At lower densities of order $\Lambda_{Q C D}^{3}$, it has been proposed that instanton generated vertices may play an important role in the Cooper pair formation. We study the renormalization group flow to the Fermi surface of the full set of couplings generated by gluonic and instanton interactions. In earlier work we showed that if the gluonic interactions dominate at the matching scale, their running determines the scale of the Cooper pair formation $\Delta$. Here we consider all possibilities, including the one in which the instanton interactions dominate all others at the matching scale. In the latter case we find that a number of additional induced couplings (including the gluonic ones) reach their Landau poles almost simultaneously with the instanton vertex. Presumably all contribute to the Cooper pair formation. The most important consequence of including all the couplings is a large increase in the gap size
\end{abstract} $\Delta$.

*nevans@budoe.bu.edu

†hsu@duende.uoregon.edu

$\ddagger$ myckola@baobab.rutgers.edu 


\section{Introduction}

Quark matter at high density exhibits color superconductivity through the dynamical generation of a Cooper pair $\left\langle\psi^{T}(-p) C \gamma_{5} \psi(p)\right\rangle$ (see [1] and references contained therein). A simple way to understand this phenomena is through an effective field theory description [2, 3] of the physics near the Fermi surface (FS). An attractive coupling between the quarks, such as that provided by one gluon exchange, will eventually run to a Landau pole as the Wilsonian cutoff approaches the FS. Of course, this is just a modern reformulation of an insight first gained from the study of laboratory superconductors [4]. At very high densities the effective theory may be matched to QCD in a naive fashion with perturbative effects like one gluon exchange dominating the dynamics [5]. However, when the Fermi momentum is reduced to of order $\Lambda_{Q C D}$ (assuming that this density is just above the transition from the low density chiral symmetry breaking phase) it is possible that additional attractive interactions exist generated by non-perturbative dynamics. An example of such a vertex is that generated by instantons (we assume there are two quark flavors throughout this paper)

$$
-\kappa\left(\bar{u}_{R} T^{a} u_{L} \bar{d}_{R} T^{a} d_{L}-\bar{u}_{R} T^{a} d_{L} \bar{d}_{R} T^{a} u_{L}\right)
$$

Recent investigations [6] examined the role of this interaction in color superconductivity under the assumption that it is the unique important coupling for the dynamics. This assumption, while an interesting starting point for studying the effects of these non-perturbative couplings, is somewhat ad hoc. Quantum loops generated by the instanton vertex produce additional interactions with different Lorentz structure which, since the couplings are of order one, we would expect to be of equal importance. In this paper we wish to study the renormalization group flow of the full set of possible couplings that close under renormalization to determine which couplings are important to the Cooper pair formation.

QCD at finite density may be described by the following lagrangian with chemical potential $\mu$

$$
\mathcal{L}=-\frac{1}{4} F^{a \mu \nu} F_{\mu \nu}^{a}+\bar{\psi}_{i}\left(i \not D+\mu \gamma_{0}\right) \psi_{i}
$$

We make a guess as to the form of the effective theory close to the Fermi surface; the obvious guess based on the dynamics of non-relativistic systems is that the theory is one of weakly interacting quarks: these are the dressed "quasi-particles" of solid state physics language. Rather than treating the gluons and instantons as dynamical degrees of freedom we will integrate them out leaving a potentially infinite sum over higher dimension fermion operators. The locality of these operators requires that gluons be screened at long-distances, presumably by effective electric and magnetic mass terms induced by the medium. An electric mass for the gluons arises at leading order in perturbation theory, and although there does not appear to be a corresponding magnetic mass (at least in perturbation theory), 
Landau damping is sufficient to prevent IR divergences due to color-magnetic fluctuations. In the region in which we are interested, where instanton effects are important, it seems likely that non-perturbative effects (for example, screening due to color-magnetic monopole excitations) will generate an effective magnetic mass. A more complete calculation would take into account possible momentum form factors in the operators, although it can easily be shown that the RG flows of components with different angular momenta decouple near the Fermi surface. For simplicity, we will therefore assume that the QCD interactions can be matched to momentum independent (s-wave) four fermion operators. We assume that the typical gauge propagator has momentum of order $\mu$. Since the Fermi surface breaks the $O(3,1)$ invariance of the theory to $O(3)$ we must treat spatial and temporal interactions independently. The full set of couplings we consider are (up to parity transformations)

$$
\begin{aligned}
& g_{1}\left(\bar{u}_{L} \gamma_{0} u_{L} \bar{u}_{L} \gamma_{0} u_{L}+u \Leftrightarrow d\right) \\
& g_{2}\left(\bar{u}_{L} \gamma_{i} u_{L} \bar{u}_{L} \gamma_{i} u_{L}+u \Leftrightarrow d\right) \\
& g_{3}\left(\bar{u}_{L} \gamma_{0} u_{L} \bar{u}_{R} \gamma_{0} u_{R}+u \Leftrightarrow d\right) \\
& g_{4}\left(\bar{u}_{L} \gamma_{i} u_{L} \bar{u}_{R} \gamma_{i} u_{R}+u \Leftrightarrow d\right) \\
& g_{5} \bar{u}_{L} \gamma_{0} u_{L} \bar{d}_{R} \gamma_{0} d_{R} \\
& g_{6} \bar{u}_{L} \gamma_{i} u_{L} \bar{d}_{R} \gamma_{i} d_{R} \\
& g_{7} \bar{u}_{L} \gamma_{0} u_{L} \bar{d}_{L} \gamma_{0} d_{L} \\
& g_{8} \bar{u}_{L} \gamma_{i} u_{L} \bar{d}_{L} \gamma_{i} d_{L} \\
& g_{9} \bar{u}_{L} \gamma_{0} d_{L} \bar{d}_{L} \gamma_{0} u_{L} \\
& g_{10} \bar{u}_{L} \gamma_{i} d_{L} \bar{d}_{L} \gamma_{i} u_{L} \\
& g_{11} \bar{u}_{L} u_{R} \bar{d}_{L} d_{R} \\
& g_{12} \bar{u}_{L} \gamma_{0} \gamma_{i} u_{R} \bar{d}_{L} \gamma_{0} \gamma_{i} d_{R} \\
& g_{13} \bar{u}_{L} d_{R} \bar{d}_{L} u_{R} \\
& g_{14} \bar{u}_{L} \gamma_{0} \gamma_{i} d_{R} \bar{d}_{L} \gamma_{0} \gamma_{i} u_{R}
\end{aligned}
$$

where the indicated groupings of couplings imply closure under RG flow. The first three groupings of couplings do not include all possible gamma matrix structures but we neglect those not present because the instanton vertex of (1) does not mix under RG flow with the one gluon vertices in these channels. The fourth grouping includes one gluon exchange in the left-left channel between two quarks of different flavors and the instanton vertex (1). RG closure of the fourth grouping requires that we include all possible gamma matrix structures. Henceforth we will include signs from the contraction of spacelike $\gamma_{i}$ matrices in the coupling constants. 
Here we will consider the $\overline{3}$ channel (which has been shown to be the most attractive channel for both the gluonic [1, 2] and instanton [6] vertices) with the color group matrix structure

$$
\delta_{c a} \delta_{d b}-\delta_{c b} \delta_{d a}
$$

At high densities, where single gluon exchange dominates the perturbative effects, the appropriate matching conditions are

$$
\begin{array}{cc}
g_{1}=g_{3}=g_{5}=g_{7}=g=\frac{1}{3} \frac{4 \pi \alpha_{s}(\mu)}{\mu^{2}} & g_{2}=g_{4}=g_{6}=g_{8}=-g \\
g_{13}=-g_{11}=\kappa & g_{9}=g_{10}=g_{12}=g_{14}=0
\end{array}
$$

where $\kappa$ is the instanton-generated four-fermion coupling considered in [6]. The sign of $\kappa$ is a result of the non-perturbative dynamics and is traditionally [6] taken positive so (1) is capable of driving chiral symmetry breaking at $\mu=0$. Since we are addressing the consistency of [6] we will do likewise.

The Fermi surface in (2) picks out momenta of order $\mu$. It is therefore natural to study the theory as we approach the Fermi surface in a Wilsonian sense. We parameterize four momenta in the following fashion

$$
p^{\mu}=\left(p_{0}, \vec{p}\right)=\left(k_{0}, \vec{k}+\vec{l}\right)
$$

where $\vec{k}$ lies on the Fermi surface and $\vec{l}$ is perpendicular to it. We study the Wilsonian effective theory of modes near the Fermi surface, with energy and momenta

$$
\left|k_{0}\right|,|\vec{l}|<\Lambda \quad, \quad \Lambda \rightarrow 0
$$

In this limit the four fermion operators are all irrelevant operators excepting those with the particular three momentum structure corresponding to quarks with momenta $\vec{k}$ and $-\vec{k}$ scattering to momenta $\vec{q}$ and $-\vec{q}[2,3]$.

As a result of this truncation of the theory the only diagrams allowed by the momentum structure are the bubble diagrams found at large $\mathrm{N}$ in the familiar $\mathrm{O}(\mathrm{N})$ model $\mathrm{W}$. To display the basic behavior, consider a theory with just the simple interaction

$$
G \bar{\psi} \psi \bar{\psi} \psi
$$

The interaction generates cooper pair formation through the exact gap equation (see Bailin and Love [1])

$$
\Delta=i G \int_{0}^{\Lambda_{U V}} \frac{d^{4} p}{(2 \pi)^{4}} \Gamma^{\mu} C(p) \Gamma_{\mu}
$$

\footnotetext{
${ }^{1}$ The expansion parameter analogous to $1 / N$ is $\Lambda / \mu[3]$.
} 
where $\mathrm{G}$ is the coupling, $\Gamma^{\mu}$ any associated Dirac structure and $C(k)$ a $4 \times 4$ off diagonal component of the $8 \times 8$ propagator associated with the fermion vector $\left(\psi, \psi^{C}\right)$

$$
C(p)=\frac{1}{\left(\not p-\mu \gamma_{0}\right)} \Delta \frac{1}{\left[\tilde{\Delta}\left(\not p-\mu \gamma_{0}\right)^{-1} \Delta-\left(\not p+\mu \gamma_{0}\right)\right]} .
$$

The gap integral would be log divergent near the FS were it not for the condensate $\Delta$, which cuts off the contribution of modes with very low energies. Thus no matter how small the (attractive) coupling $G$ there will always be a solution for some $\Delta$. Approximating the IR cutoff, and keeping the lowest order in $\Delta$ we have

$$
\Delta \sim-i G \int_{\Delta}^{\Lambda_{U V}} \frac{d^{4} p}{(2 \pi)^{4}} \frac{1}{\left(\not p-\mu \gamma_{0}\right)} \Delta \frac{1}{\left(\not p+\mu \gamma_{0}\right)},
$$

Neglecting factors from the gamma matrix structure, the Cooper pair condensate is of the form

$$
\Delta=\Lambda_{U V} e^{-\frac{c}{N G}}
$$

where $c$ is a constant.

An alternative understanding of this condensate formation is found by resumming bubble graphs to calculate the renormalization group flow of the four quark vertex. Note that because of the restricted momentum structure of the vertices one loop $\beta$ functions are exact. The one loop graph contributes a logarithmic divergence to the running in the presence of a Fermi surface:

$$
-G^{2} \int \frac{d^{4} p}{(2 \pi)^{4}}\left[\frac{i}{p^{\mu} \gamma_{\mu}+\mu \gamma_{0}-i \epsilon}\right]_{i k}\left[\frac{i}{-p^{\nu} \gamma_{\nu}+\mu \gamma_{0}-i \epsilon}\right]_{j l}
$$

Performing the gamma matrix algebra and taking the limit $k_{0},|\vec{l}| \rightarrow 0,|\vec{k}|^{2} \rightarrow \mu^{2}$, this becomes

$$
G^{2}\left[-\left(\gamma_{0}\right)_{i j}\left(\gamma_{0}\right)_{k l}+\frac{1}{3}\left(\gamma_{\alpha}\right)_{i j}\left(\gamma_{\alpha}\right)_{k l}\right] I
$$

where the log divergent part of the integral I is given by

$$
\begin{aligned}
I & =\frac{1}{4} \int \frac{d k_{0} d^{2} k d l}{(2 \pi)^{4}} \frac{1}{\left(k_{0}+l-i \epsilon\right)\left(k_{0}-l+i \epsilon\right)} \\
& \simeq \frac{i}{4} N \ln \left(\frac{\Lambda_{I R}}{\Lambda_{U V}}\right) .
\end{aligned}
$$

$N=\int d^{2} k /(2 \pi)^{3}=\mu^{2} / 2 \pi^{2}$, assuming the density of states at the Fermi surface is given by the lowest order approximation. The running effective coupling (neglecting the gamma matrix structure again) is given by

$$
G\left(\Lambda_{I R}\right)=\frac{G\left(\Lambda_{U V}\right)}{1+\frac{1}{4} G\left(\Lambda_{U V}\right) N t}
$$


where $t=\ln \left(\Lambda_{I R} / \Lambda_{U V}\right)$. Here we have moved from an effective theory with cutoff $\Lambda_{U V}$ $\left(k_{0},|\vec{l}|<\Lambda_{U V}\right)$, to a new effective theory with cutoff $\Lambda_{I R}$. As we approach the Fermi surface $\left(\Lambda_{I R} \rightarrow 0\right)$, the coupling $G$ runs logarithmically. The Landau pole of the coupling corresponds to the scale $\Delta$ of Cooper pair formation.

In this fashion we can calculate the one loop beta functions for the vertices in (3) as a function of $t$. There are in principle $14^{2}$ entries to the RG matrix but many entries are trivially zero. The RG equations simplify drastically if one introduces the linear combinations of couplings

$$
\begin{array}{lll}
G_{1}=g_{1}+g_{2} & G_{7}=g_{7}+g_{8} & G_{11}=g_{11}+g_{12} \\
G_{2}=g_{1}-3 g_{2} & G_{8}=g_{7}-3 g_{8} & G_{12}=g_{11}-3 g_{12} \\
G_{3}=g_{3}-g_{4} & G_{9}=g_{9}+g_{10} & G_{13}=g_{13}+g_{14} \\
G_{4}=g_{3}+3 g_{4} & G_{10}=g_{9}-3 g_{10} & G_{14}=g_{13}-3 g_{14} \\
G_{5}=g_{5}-g_{6} & & \\
G_{6}=g_{5}+3 g_{6} & &
\end{array}
$$

and the RG equations then simplify to

$$
\begin{array}{lll}
\dot{G}_{1}=-\frac{1}{3} N G_{1}^{2} & \dot{G}_{7}=-\frac{1}{3} N\left(G_{7}^{2}+G_{9}^{2}+G_{11}^{2}+G_{13}^{2}\right) & \dot{G}_{11}=-\frac{2}{3} N\left(G_{7} G_{11}+G_{9} G_{13}\right) \\
\dot{G}_{2}=-N G_{2}^{2} & \dot{G}_{8}=-N\left(G_{8}^{2}+G_{10}^{2}+G_{12}^{2}+G_{14}^{2}\right) & \dot{G}_{12}=-2 N\left(G_{8} G_{12}+G_{10} G_{14}\right) \\
\dot{G}_{3}=-\frac{2}{3} N G_{3}^{2} & \dot{G}_{9}=-\frac{2}{3} N\left(G_{7} G_{9}+G_{11} G_{13}\right) & \dot{G}_{13}=-\frac{2}{3} N\left(G_{7} G_{13}+G_{9} G_{11}\right) \\
\dot{G}_{4}=\dot{G}_{6}=0 & \dot{G}_{10}=-2 N\left(G_{8} G_{10}+G_{12} G_{14}\right) & \dot{G}_{14}=-2 N\left(G_{10} G_{12}+G_{8} G_{14}\right) \\
\dot{G}_{5}=-\frac{2}{3} N G_{5}^{2} & &
\end{array}
$$

We proceed further by numerical solution of the RG equations. At very high density the instanton vertex is expected to be exponentially suppressed relative to the one gluon exchange. Neglecting the instanton vertex removes any flavor dependence from the problem and there are just four independent couplings, the spatial and temporal couplings between two left handed quarks and between a left and a right handed quark. We show the RG running in Fig 1. The coupling between two left handed quarks reaches its Landau pole first as found in [2].

We may check the effect of the instanton by including it but with a smaller value than the one gluon exchange at the matching scale. We show this case in Figure 2 with $\kappa=0.01 g$. The gluonic couplings reach their Landau pole before the instanton vertex becomes large, though the instanton vertex is eventually driven to infinity by feedback from the larger gluon interaction. When we include the full RG flow the instanton vertex does eventually catch the gluonic coupling but only at very large values of the coupling $\gg 10$, which increase for smaller values of the instanton coupling at matching. The scale of the Landau pole, 
and hence the size of the Cooper pairing gap, is determined by the running of the gluonic couplings as expected.

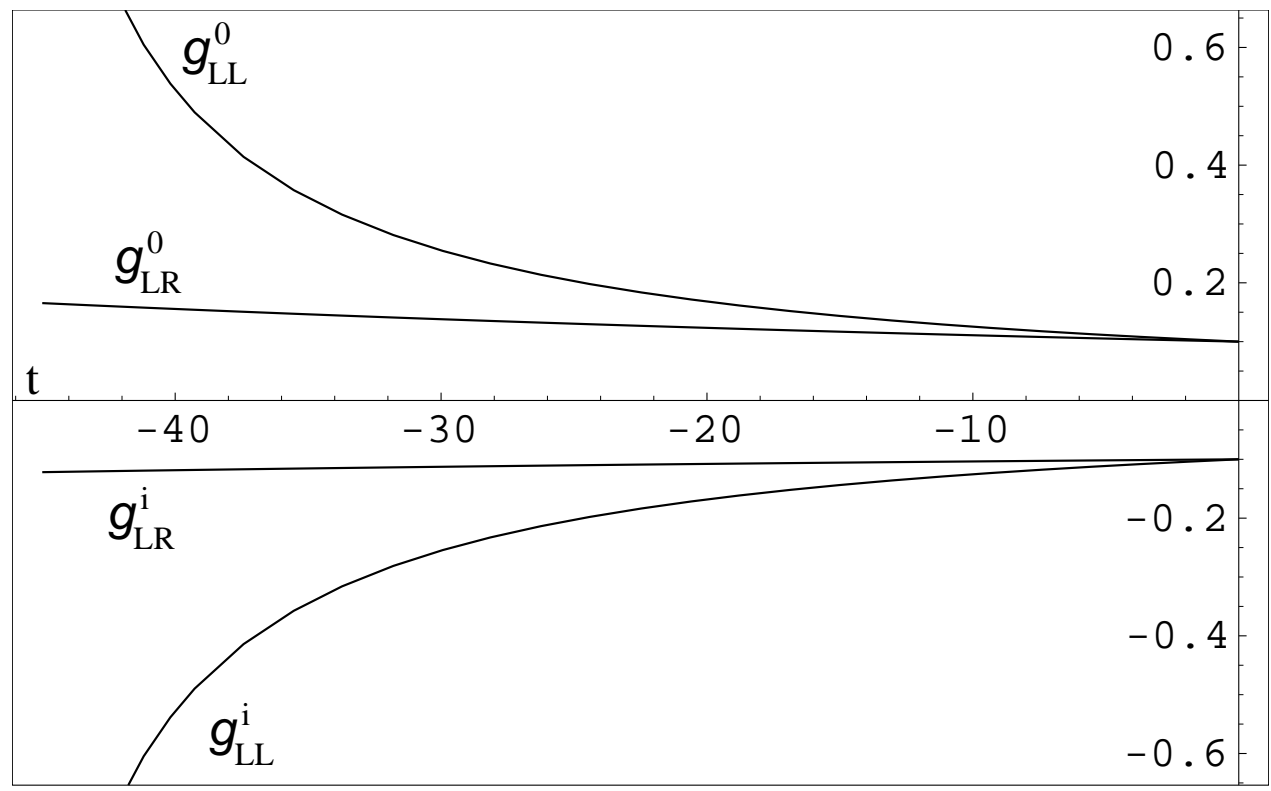

Figure 1: The RG running of the temporal and spatial one gluon derived couplings between two left handed quarks and that between a left handed and a right handed quark in the absence of instanton effects.

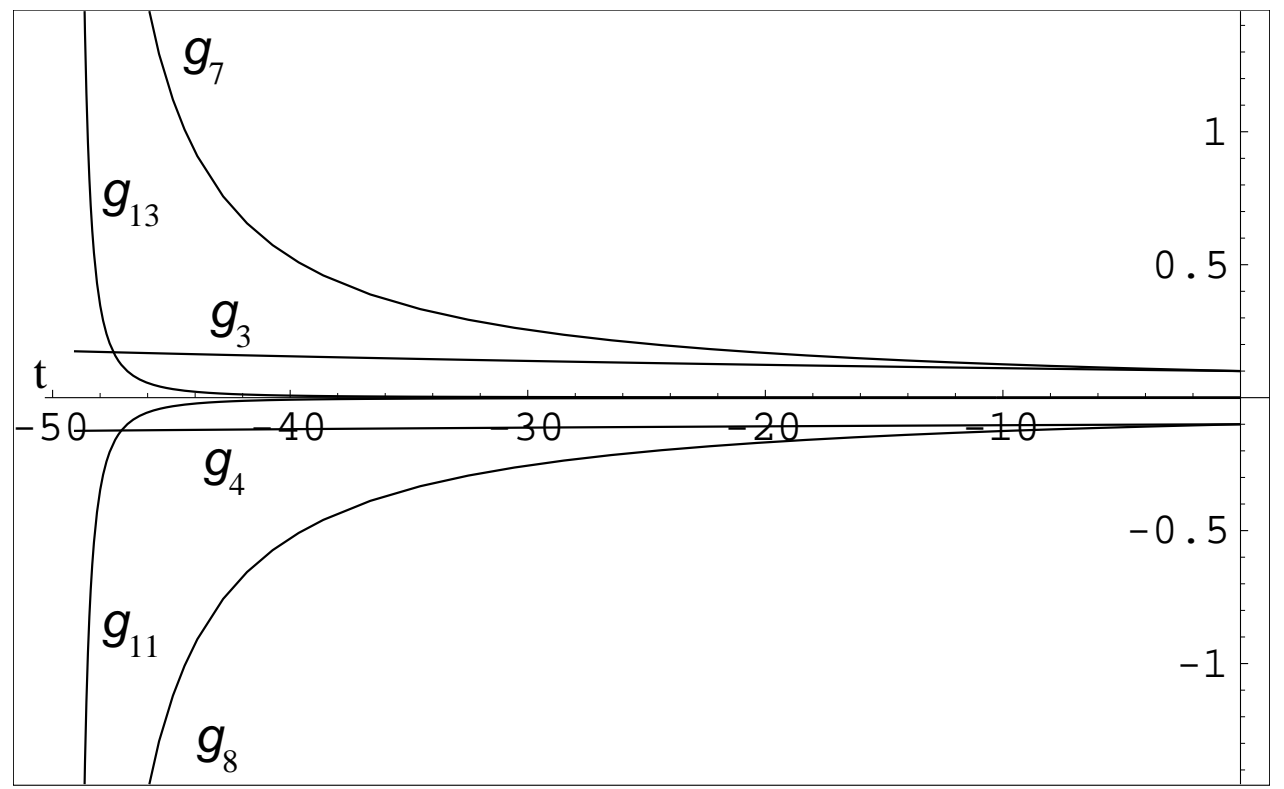

Figure 2: The influence of a small instanton coupling $\left(g_{11}\right.$ and $\left.g_{13}\right)$ on the running of the gluonic couplings between an up quark and a down quark $\left(g_{7}\right.$ and $g_{8}$ are in the left left channel, $g_{3}$ and $g_{4}$ in the left right channel). 
We next turn to the effects of the instanton vertex when it is the dominant interaction. It is interesting to see what happens in the case where the instanton vertex is treated as the sole non-zero coupling at the matching scale. This scenario is shown in Fig 3. The 8 couplings $\left(g_{7}-g_{14}\right)$ all reach values of order one simultaneously and presumably play an equal role in Cooper pair formation. This is not a surprise since, as can be seen from (18), the instanton couplings $G_{11}-G_{14}$ only run after the generation of couplings $G_{7}-G_{10}$. Note that for a very thin shell around the FS $\left(\Lambda_{U V}<<\mu\right)$ this running is implicitly included in a gap equation analysis performed with just the instanton coupling at the matching scale, since the gap equation is exact in this limit. What this analysis shows is that as expected the instanton coupling, which generates the other vertices at one loop, drives them rather quickly to large values. The analysis we have performed retains only the relevant operators near the FS. The effects of the intermediate running (including irrelevant operators) which takes us close to the FS is assumed to be reflected in our boundary conditions at the matching scale. Our results suggest that in QCD at low densities (of order $\Lambda_{Q C D}^{3}$ ), where the instanton coupling $\kappa$ is of order one, the correct analysis would include all 8 couplings with approximately equal values at matching.

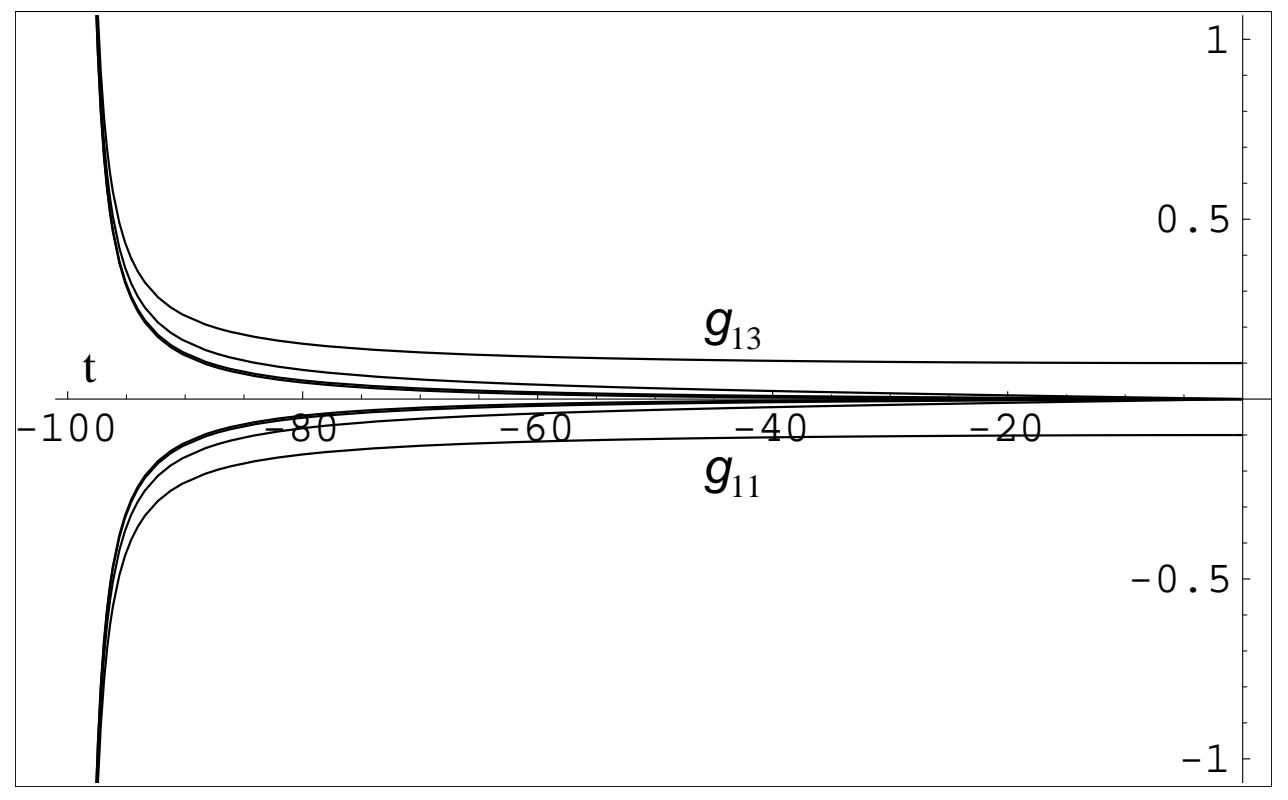

Figure 3: A toy model in which the instanton couplings $\left(g_{11}\right.$ and $\left.g_{13}\right)$ are the only non-zero coupling at the matching scale. The other six couplings grow from zero $\left(g_{7}, g_{10}, g_{12}\right.$ have positive values, $g_{8}, g_{9}, g_{14}$ negative) and reach Landau poles at approximately the same scale as the instanton vertices.

If we do assume that all eight interactions are equal at the matching scale and have the natural choices of sign suggested by Fig 3 then the RG equations simplify. The couplings 
$G_{\text {odd }}$ taken with initial conditions 0 remain 0 for the full running. The couplings $G_{\text {even }}$ with initial conditions $\pm 4 G$ run with the same absolute values and share the same Landau pole. In terms of the orginal 8 couplings this means that they all run together and reach their Landau poles simultaneously. All possible interactions appear to play an equal role in the Cooper pair formation. We show the running in Fig 4.

The Cooper pair formation with only an instanton interaction at matching has been studied in [6] and for the two flavor case the condensate that forms is in the anti-symmetric color $\overline{3}$, is an anti-symmetric singlet in flavor, and an anti-symmetric singlet of spin (as is the case when the condensate is driven by gluonic interactions [1]). Since for the instanton case, as can be seen from Fig 3 , all the couplings $g_{7}-g_{14}$ are essentially equal at the Landau pole we may deduce that for the case where this equality is enforced at the matching the same condensate forms. The important difference is that the scale of the Landau pole is increased sharply by the inclusion of the full set of couplings at matching. Typically, whatever the matching condition taken on the couplings, the value of $|t|$ at the pole is significantly smaller than for the pure instanton case. The Cooper pair condensate, if non-perturbative effects are sufficiently large to play a role, is therefore expected to be considerably larger than the estimates including only the instanton vertex.

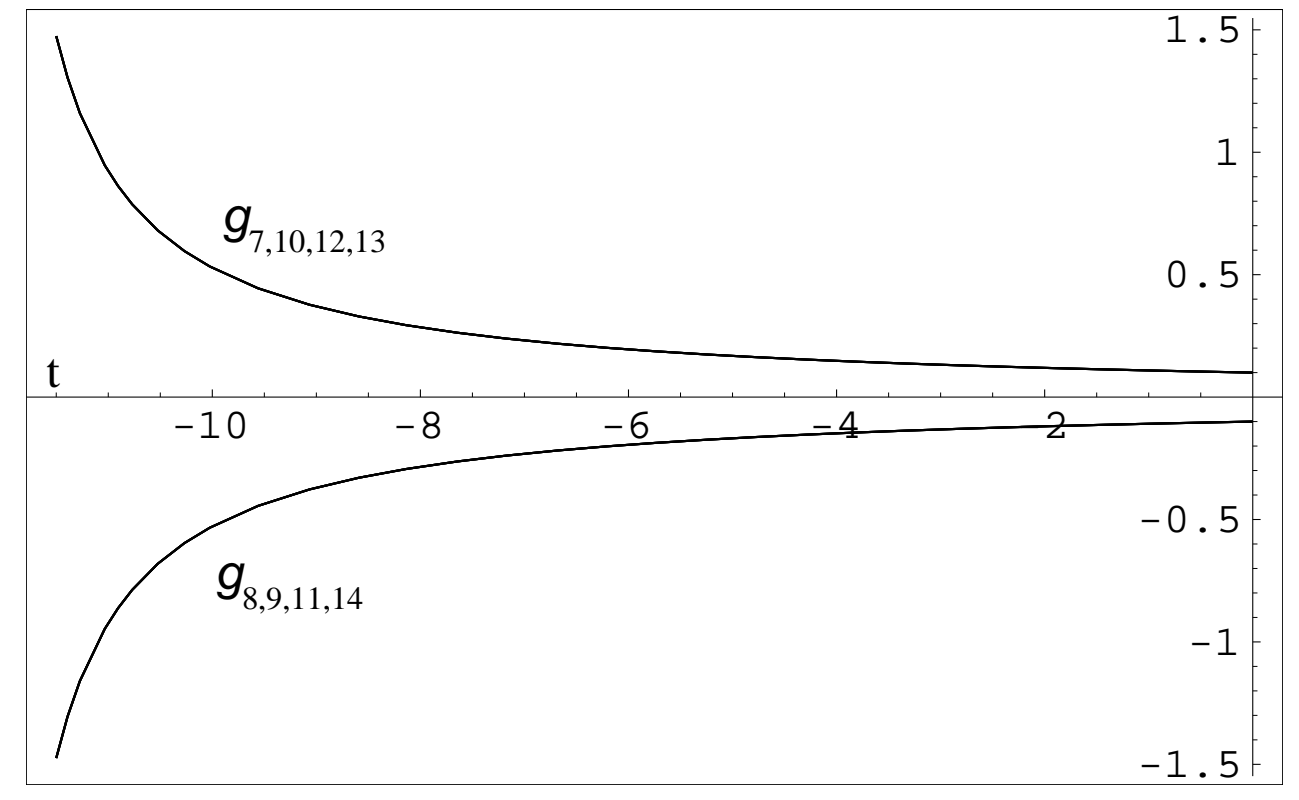

Figure 4: The RG flow when all 8 couplings interacting with the instanton vertex $\left(g_{7}-g_{14}\right)$ are taken equal at matching. The couplings evolve together. 
The authors would like to thank M. Alford, M. Peskin, K. Rajagopal, T. Schafer and F. Wilczek for useful discussions and comments. After this work was completed we became aware of work by Schafer and Wilczek (IASNS-HEP-98-90) which addresses similar issues. Their analysis includes a simplification of the RG equations through the use of flavor symmetries and Fierz identities. This work was supported in part under DOE contracts DEFG02-91ER40676, DE-FG06-85ER40224 and DE-FG02-96ER40559

\section{References}

[1] D. Bailin and A. Love, Nucl. Phys. B190 (1981) 175; Nucl. Phys. B190 (1981) 751; Nucl. Phys. B205 (1982) 119; Phys. Rep. 107 (1984) 325.

[2] N. Evans, S.D.H. Hsu and M. Schwetz, hep-ph/9808444.

[3] G. Benfatto and G. Gallavotti, J. Stat. Phys. 59, 541 (1990); Phys. Rev. C42 (1990) 9967; R. Shankar, Physica A177, 530 (1991); Rev. Mod Phys. 66, 129 (1993); J. Polchinski, in Proceedings of the 1992 TASI, eds. J. Harvey and J. Polchinski (World Scientific, Singapore 1993).

[4] See, for example, N. Ashcroft and N.D. Mermin, Solid State Physics, Saunders College Publishing (1976).

[5] Freedman and McLerran, Phys. Rev. D16 (1977) 1130; Phys. Rev. D16 (1977) 1147; Phys. Rev. D16 (1977) 1169.

[6] R. Rapp, T. Schafer, E.V. Shuryak and M. Velkovsky, Phys. Rev. Lett. 81 (1998) 53; M. Alford, K. Rajagopal and F. Wilczek, Phys. Lett. B422 (1998) 247; M. Alford, K. Rajagopal and F. Wilczek, hep-ph/9804403. 